\title{
Construindo um observatório nacional da judicialização da política pública de saúde a partir dos municípios brasileiros: desenho de estudo
}

Developing a national observatory on the judicialization of public health policy in Brazilian municipalities: study design

Desarrollando un observatorio nacional de la judicialización de la política pública de salud de los municipios brasileños: diseño de estudio

Pedro Paulo Chrispim ${ }^{1}$

Mayla Youko Kato ${ }^{1}$

Nicoli Prado Bugoni ${ }^{1}$

Gizelda Monteiro da Silva ${ }^{1}$

Rosangela Bogues Pittioni ${ }^{1}$

Bernardete Weber ${ }^{1}$

RESUMO. Objetivo: O artigo descreve o percurso metodológico utilizado na construção de um levantamento de dados como pesquisa exploratória com amostragem sistemática para se conhecer a natureza das ações judiciais (contra) em que figuram como réu, os governos municipais que demandam produtos e serviços para a Saúde. Metodologia: O projeto foi planejado para ser desenvolvido no âmbito do Programa de Apoio ao Desenvolvimento Institucional do Sistema Único de Saúde como parceria entre a Fundação Oswaldo Cruz de Brasília, o Ministério da Saúde e o Hospital do Coração em São Paulo e cobrir todo o território brasileiro como um esforço para dimensionar um quadro nacional da Judicialização das Políticas Públicas de Saúde nos municípios e subsidiar a formação de um Observatório Nacional da Judicialização da Saúde. Resultados: Os resultados devem contribuir para um melhor controle das demandas judiciais tanto por parte do Ministério da Saúde como por parte dos Tribunais de Justiça por meio do conhecimento das demandas em si e dos caminhos percorridos pelos autores das ações para a formação dos processos. Conclusão: O projeto cobre uma lacuna de conhecimento importante sobre a natureza das demandas judiciais e políticas públicas de saúde e propõe o exercício da continuidade do monitoramento das demandas judiciais, que crescem dia após dia e se especializam em diversos aspectos.

Palavras-chave: Judicialização da Saúde. Métodos. Saúde Pública.

ABSTRACT. Objective: This article describes the methodological course used in the construction of a data survey as exploratory research with systematic sampling to know the nature of the lawsuits against municipal governments that demand products and services for Health. Methodology: The project was planned to be developed in the scope of the Program of Support for the Institutional Development of the Unified Health System as a partnership between the Oswaldo Cruz Foundation of Brasilia, the Ministry of Health and the Heart Hospital in São Paulo and cover the entire Brazilian territory as an effort to dimension a national framework of Judicialization of the Public Health Policies in the municipalities and to subsidize the formation of a National Observatory of the Judicialization of Health. Results: Its results should contribute to a better control of the judicial demands both by the Ministry of

\footnotetext{
${ }^{1}$ Hospital do Coração. E-mail: pedropaulo.chrispim@gmail.com
} 
Health and by the Courts of Justice through the knowledge of the demandsin itself and of the paths taken by the authors of the actions for the formation of the processes. Conclusion: The project covers a gap of important knowledge about the nature of the lawsuits and public health policies, and proposes the exercise of continuity of monitoring of lawsuits, which grow day after day, and specialize in several aspects.

Keywords: Judicialization of Health. Methodology. Public Health.

RESUMEN. Objetivo: El artículo describe el recorrido metodológico utilizado en la construcción de una recopilación de datos como investigación exploratoria con muestreo sistemático para conocer la naturaleza de las acciones judiciales contra gobiernos municipales que demandan productos y servicios para la Salud. Metodología: El proyecto fue planeado para ser desarrollado en el ámbito del Programa de Apoyo al Desarrollo Institucional del Sistema Único de Salud como una colaboración entre la Fundación Oswaldo Cruz de Brasilia, el Ministerio de Salud y el Hospital del Corazón en Sao Paulo, y para cubrir todo el territorio brasileño como un esfuerzo para dimensionar un marco nacional de la Judicialización de las Políticas Públicas de Salud en los municipios y subsidiar el desarrollo de un Observatorio Nacional de la Judicialización de la Salud. Resultados: Los resultados deben contribuir a un mejor control de las demandas judiciales tanto por parte del Ministerio de Salud como de los Tribunales de Justicia a través del conocimiento de las demandas y de los caminos recorridos por los demandantes para la apertura de los procesos. Conclusión: El proyecto cubre un vacío importante de conocimiento sobre la naturaleza de las acciones judiciales y las políticas de salud pública, y propone el ejercicio de la continuidad de la vigilancia de las demandas judiciales, que crece día tras día, y se especializan en diversos aspectos.

Palabras-Ilave: Judicialización de la Salud. Metodología. Salud pública.

\section{Introdução}

Os procedimentos judiciais envolvendo interesses individuais e coletivos vinculados ao Direito à Saúde têm tido seu número ampliado significativamente no judiciário brasileiro, ocupando crescente participação no comprometimento financeiro e orçamentário nas diversas instâncias de gestão do Sistema Único de Saúde (SUS). Este cenário tem suscitado a necessidade de maior atenção por parte dos gestores do SUS, melhor entendimento de como este fenômeno tem ocorrido, diversas motivações dos elementos vinculados ao processo, para com isso, ampliar a capacidade de resposta do SUS e da Justiça a essa dinâmica.

Se a judicialização das Políticas Públicas de Saúde no Brasil não é exatamente um mistério, tampouco se pode dizer que a conhecemos profundamente. Existem, certamente, muitos estudos sobre o tema que contribuem para conhecermos como se dá a judicialização e quais ações judiciais que demandam importante resultado orçamentário e colocam em questão o equilíbrio entre o direito individual e o coletivo (1) (2) (3) (4) (5) (6) (7) (8) (9). 
Outra característica importante nos estudos sobre judicialização das Políticas Públicas de Saúde no país é a metodologia que as define. Parecem, via de regra, contingenciadas pela dinâmica atual do financiamento e prestação de contas das pesquisas financiadas com recursos públicos em duas diversas modalidades; seja pelo próprio tempo do pesquisador responsável ou seus agentes de campo, que se encontram em uma instituição pública, seja pelo financiamento direto das agências de fomento. Em sua maior parte, é na coleta de dados que se verifica o que pode ser a principal consequência desse contingenciamento: 0 maior volume de produção de pesquisas utiliza a facilidade oferecida pelos tribunais informatizados ou se restringe a um recorte específico que delimita a quantidade de dados a serem coletados. O foco principal dessas pesquisas têm sido as demandas judiciais por medicamentos (9) (10) (11) (12) (13) (14). Isso é justo e legítimo, uma vez que são as demandas que resultam em maior contingenciamento orçamentário e estão no centro da discussão entre os limites do direito individual e do coletivo (15).

Algumas iniciativas têm buscado ampliar o conhecimento sobre o universo de ações e isso tem se dado principalmente como resultado das iniciativas do CNJ (Conselho Nacional de Justiça) que tem publicado resoluções específicas para lidar com o aumento das ações sobre a Saúde. A criação do Fórum Nacional da Saúde é, provavelmente, a iniciativa mais representativa e que determinou a instrução processual e apontou caminhos para que os juízes tivessem maior apoio às suas decisões, principalmente no que tange à elevada tecnicalidade e especificidade do tema (16). Houve um crescimento das ações com demandas em Saúde e o crescimento de núcleos de apoio técnico aos juízes e às decisões em saúde, corporificados nos Núcleos de Apoio Técnico à justiça ou, como comumente chamados NAT-jus (17) além de crescerem, os trabalhos entre tribunais e secretarias de saúde também aumentam em número e representatividade, núcleos são criados a fim de darem suporte às decisões judiciais e a formação de acordos antes de as ações se tornarem litigiosas (18) (19).

Todas essas iniciativas, porém, nos fornecem uma visão fragmentada do universo de ações na justiça e frequentemente focadas onde há facilidade de acesso aos dados, o que, no momento atual, resulta em dados das Regiões Sul e Sudeste do Brasil, onde há maior cobertura de sistemas informatizados para o trâmite processual. Consequentemente, os responsáveis por consolidar as informações atuam centralmente, coletando dados via internet em seus locais de residência profissional, sem necessidade de deslocamento para 
o tribunal em que os processos fisicamente estão (20). Se por um lado, isso auxilia no conhecimento desses processos, de outro, fragmenta o conhecimento do estágio atual do conjunto de ações movidas na justiça contra as Políticas Públicas de Saúde em todo o Brasil.

Outra lacuna de informação a ser preenchida está no fato de que mesmo as pesquisas mais abrangentes realizadas até hoje foram justamente fruto das iniciativas do Conselho Nacional de Justiça (CNJ), o que fornece respostas às questões sobre acesso e direito à Saúde, mas não necessária e sistematicamente às questões de Saúde, em si (20).

Nessa lacuna de informação encontrou-se justificativa para a idealização e execução de um projeto que almejasse desenhar a coleta de dados em todo o território nacional, para conhecer as motivações e demandas em Saúde no Brasil, de forma representativa de modo a tornar habitual essa coleta de informações que tem o objetivo de subsidiar tanto 0 Ministério da Saúde em seu planejamento preventivo (e corretivo) em Saúde quanto o Ministério da Justiça sobre as instruções processuais e ambos, para informá-los sobre como se distribuem, no país, as ações judiciais que representam as dificuldade de acesso à Saúde da população brasileira.

\section{Metodologia}

Tratou-se de um estudo exploratório de amostragem sistemática cujo principal objetivo foi conhecer a distribuição de ações judiciais contra as Políticas Públicas de Saúde sentenciadas (julgadas) em primeira instância em todo o território nacional. Sua origem residiu no trabalho que o Programa de Direito Sanitário (Prodisa) da Fiocruz Brasília realizou na coleta de dados de ações judiciais contra os estados entre os anos de 2012 e 2013. Nesse trabalho, procurou-se conhecer a natureza das ações existentes no país por meio dessas ações que existiam nos Tribunais das 26 capitais brasileiras e do Distrito Federal. A expansão natural dessa pesquisa inicial seria a coleta de dados sobre as ações judiciais contra os governos municipais em todo o território nacional e com isso ter-se um quadro mais abrangente das demandas judicializadas.

O delineamento do estudo foi decidido em comitê composto pela Fiocruz Brasília, HCor e Secretaria Executiva do Ministério da Saúde, que decidiram conjuntamente sobre o formulário de coleta de dados, e a amostragem das comarcas a serem visitadas.

A execução do projeto foi coordenada centralmente pelo HCor, responsável pela execução financeira e coordenação de pesquisadores. A coleta de dados foi coordenada de 
forma descentralizada e multicêntrica com equipes de coleta de dados nas cinco regiões brasileiras.

A produção dos relatórios de pesquisa foi de responsabilidade do HCor e publicações científicas provenientes das análises de dados serão de responsabilidade compartilhada entre os três integrantes institucionais do comitê que delineou o estudo, sendo possível a iniciativa individual ou compartilhada das instituições para propor análises dos dados e suas respectivas publicações.

A simples expansão do projeto inicial, entretanto, não contemplaria questões que vêm ganhando importância para os gestores do Ministério da Saúde. Questões como o crescente desequilíbrio entre o direito particular e o direito social ou sobre os papéis que desempenham os poderes Executivo e o Judiciário (6), constantemente sobrepostos no caso de algumas ações cujo resultado financeiro sobrecarregam o Ministério da Saúde e têm o potencial de comprometer os Programas Assistenciais existentes.

Fora definido, portanto, este projeto como uma forma de amostragem sistemática que pudesse captar dados de interesse mais amplo em todas as comarcas que viessem a ser visitadas. Os critérios para a seleção dos processos capturados e a rotina de trabalho também foram definidos.

\section{Amostragem}

A fim de servir de parâmetro para o cálculo amostral do quantitativo de processos que deveriam ser coletados, os trabalhos mais abrangentes encontrados foram fruto de uma pesquisa conjunta entre o CNJ e o Laboratório de Pesquisas sobre Práticas de Integralidade em Saúde (LAPPIS/UERJ) e o monitoramento das ações em Saúde, resultado da Resolução no 07/2009 do CNJ (17) (21). Ambas as pesquisas não forneceram dados o suficiente, apenas os totais de processos por Tribunal Regional Federal ou dos tribunais de Justiça das capitais, não discriminando comarcas, nem o total de ações por instância, nem deixando clara a metodologia para o cálculo dos totais por estado (17). O foco do trabalho de Asensi e Pinheiro (21), por sua vez, é a resolução processual da demanda jurídica cujo volume crescente desde o início da década de 2010 vinha desequilibrando a rotina de trabalho de juízes do país inteiro (21). Os dados sobre a assistência pública e a Saúde Suplementar foram publicados em anexos posteriores, no ano seguinte (20) (21) (22). Segundo o relatório de Asensi e Pinheiro (21), focado no trabalho dos tribunais de São Paulo, Paraná, Rio 
Grande do Norte, Minas Gerais, Mato Grosso do Sul e Acre, durante os anos de 2011 e 2012, a amostra foi escolhida por conveniência para se adequar à disponibilidade de recursos para realizar a pesquisa. Dados prévios, sobre o estado geral do estoque de processos, informam que nos anos de 2011 e 2012 havia pouco mais de duas dezenas de milhões de processos judiciais em tramitação no país, (21), porém apenas alguns poucos milhares referentes ao Sistema de Saúde (20) (22). O monitoramento da Resolução 07/2009 apontou mais de 330 mil ações no conjunto dos tribunais estaduais (17).

Queria-se, entretanto, uma amostragem direta das ações julgadas em primeira instância nas comarcas, que são a unidade geomorfofuncional da organização do sistema de justiça brasileiro e para isso, não foram encontrados dados adequados. Partindo dessa incerteza sobre o quantitativo dos processos judiciais no país e quase absoluta obscuridão sobre a distribuição das demandas nas comarcas, optou-se por estimar o tamanho da amostra em duas etapas. Inicialmente, pelo número total de processos a serem capturados no campo no período disponível, que fora calculado a partir da capacidade média de coleta de dados estimada com dados da pesquisa anterior efetuada pela equipe do Prodisa.

Em seguida, foi distribuído esse total de processos a ser capturado de forma proporcional pelas comarcas existentes de maior representatividade populacional que fossem sorteadas, por agrupamento populacional, equilibrando o número total de comarcas a serem visitadas pela capacidade de visitá-las, de fato. Por "capacidade de visitar a comarca" deve ser entendido tanto o deslocamento geográfico para as visitas que fossem fisicamente necessárias, quanto a eventual substituição das comarcas para as quais o acesso aos dados das ações fosse negado. Ao fim dessa seleção, foram coletados dados de 3.679 processos em comarcas cujos municípios tinham mais de 500mil habitantes, 3.251 processos em comarcas cujos municípios-sede tinham entre 100mil e 500mil habitantes e, finalmente, 1.462 processos em comarcas cujos município-sede tinham entre 50mil e 100mil habitantes.

Existem cerca de 600 comarcas no país e há uma grande diversidade de rotinas de trabalho e formas de registro de dados, havendo tramitações completamente informatizadas e muitas outras comarcas que ainda operam sem nenhum sistema de informação para suporte aos atores do Sistema Judiciário. Tem-se, por fim, a amostra estimada em número de processos a serem capturados em cada comarca onde a coleta de dados deve ocorrer. Nota-se que para esse cálculo há o pressuposto que a distribuição das demandas é 
proporcionalmente homogênea entre as comarcas e correlacionado com sua população residente. É razoável supor que haja falhas nessa suposição, o que não temos como verificar de forma precisa sem uma pesquisa censitária. Por esse motivo optou-se por uma amostragem sistemática. A lista das comarcas a serem visitadas se encontra nas tabelas de 1 a 5.

Tabela 1. Comarcas a serem visitadas na Região Sul, os municípios cobertos pelas comarcas e a população residente em cada município.

\begin{tabular}{|c|c|c|}
\hline COMARCA & MUNICÍPIO & № HABITANTES \\
\hline \multirow{26}{*}{ 01)Porto Alegre/RS } & 1) Sede & 1.481 .019 \\
\hline & 2)Alvorada & 207.392 \\
\hline & 3)Cachoeirinha & 126.666 \\
\hline & 4)Eldorado do Sul & 38.199 \\
\hline & 5)Triunfo & 28.084 \\
\hline & 6)Viamão & 252.872 \\
\hline & 7)Campo Bom & 64.553 \\
\hline & 8)Estância Velha & 46.877 \\
\hline & 9)Esteio & 84.114 \\
\hline & 10)Gravataí & 273.742 \\
\hline & 11)Guaiba & 99.186 \\
\hline & 12)Novo Hamburgo & 249.113 \\
\hline & 13)São Leopoldo & 229.678 \\
\hline & 14)São Sebastião do Caí & 24.825 \\
\hline & 15)Sapiranga & 79.946 \\
\hline & 16)Sapucaia do Sul & 138.933 \\
\hline & 17)Viamão & 252.872 \\
\hline & 18)Dois Irmãos & 30.437 \\
\hline & 19)Glorinha & 7.588 \\
\hline & 20)Ivoti & 22.270 \\
\hline & 21)Nova Hartz & 20.225 \\
\hline & 22)Parobé & 55.893 \\
\hline & 23)Portão & 34.353 \\
\hline & 24)Triunfo & 28.084 \\
\hline & 25)Charqueadas & 38.599 \\
\hline & 26)Araricá & 5.349 \\
\hline
\end{tabular}




\begin{tabular}{|c|c|c|}
\hline & 27)Nova Santa Rita & 26.086 \\
\hline & 28)Montenegro & 63.551 \\
\hline & 29)Taquara & 57.396 \\
\hline & 30)São Jerônimo & 23.649 \\
\hline & 31)Arroio dos Ratos & 20.162 \\
\hline & 32)Santo Antônio da Patrulha & 42.160 \\
\hline & 33)Capela de Santana & 11.375 \\
\hline & 34)Rolante & 20.819 \\
\hline & 35)Igrejinha & 34.630 \\
\hline \multirow[t]{3}{*}{ 02)Canoas/RS } & 36)Sede & 342.634 \\
\hline & 37)Nova Santa Rita & 26.086 \\
\hline & 38)Esteio & 84.114 \\
\hline \multirow[t]{6}{*}{ 03)São José/SC } & 39)Sede & 228.561 \\
\hline & 40)São Pedro de Alcântara & 5.139 \\
\hline & 41)Antonio Carlos & 7.906 \\
\hline & 42)Biguaçu & 63.440 \\
\hline & 43)Palhoça & 137.334 \\
\hline & 44)Santo Amaro da Imperatriz & 21.910 \\
\hline \multirow[t]{4}{*}{$\begin{array}{l}\text { 04)Balneário } \\
\text { Camboriú/SC }\end{array}$} & 45)Sede & 124.557 \\
\hline & 46)Camboriú & 62.289 \\
\hline & 47)Itajaí & 208.958 \\
\hline & 48)Itapema & 45.814 \\
\hline \multirow[t]{7}{*}{ 05)Colombo/PR } & 49)Sede & 215.242 \\
\hline & 50)Rio Branco do Sul & 30.662 \\
\hline & 51)Bocaiuva do Sul & 11.005 \\
\hline & 52)Pinhais & 128.256 \\
\hline & 53)Quatro Barras & 19.786 \\
\hline & 54)Campina Grande do Sul & 38.756 \\
\hline & 55)Almirante Tamandaré & 112.870 \\
\hline \multirow[t]{5}{*}{$\begin{array}{l}\text { 06)São José dos } \\
\text { Pinhais/PR }\end{array}$} & 56)Sede & 292.934 \\
\hline & 57)Tijucas do Sul & 14.526 \\
\hline & 58)Fazenda Rio Grande & 81.675 \\
\hline & 59)Guaratuba & 34.920 \\
\hline & 60)Mandirituba & 22.235 \\
\hline
\end{tabular}




\begin{tabular}{|l|l|l|}
\hline & 61)Morretes & 15.718 \\
\hline & 62)Piraquara & 102.798 \\
\hline
\end{tabular}

Tabela 2. Comarcas a serem visitadas na Região Sudeste, os municípios cobertos pelas comarcas e a população residente em cada município.

\begin{tabular}{|c|c|c|}
\hline COMARCA & MUNICIPIO & № HABITANTES \\
\hline $\begin{array}{l}\text { 07)Grande São Paulo } \\
\text { l/SP }\end{array}$ & 63)Mauá & 457.696 \\
\hline & 64)Diadema & 406.418 \\
\hline & 65)Ribeirão Pires & 113.068 \\
\hline & 66)Rio Grande da Serra & 48.302 \\
\hline & 67)Santo André & 712.749 \\
\hline & 68)São Bernardo do Campo & 822.242 \\
\hline & 69)São Caetano do Sul & 158.825 \\
\hline \multirow[t]{6}{*}{$\begin{array}{l}\text { 08)Grande São Paulo } \\
\text { II/SP }\end{array}$} & 70)Mogi das Cruzes & 429.321 \\
\hline & 71)Biritiba-Mirim & 31.158 \\
\hline & 72)Mairiporã & 93.981 \\
\hline & 73)Arujá & 83.939 \\
\hline & 74)Barueri & 264.935 \\
\hline & 75)Pirapora de Bom Jesus & 17.646 \\
\hline \multirow{11}{*}{$\begin{array}{l}\text { 09) Santana de } \\
\text { Parnaíba/SP }\end{array}$} & 76)Santana de Parnaíba & 129.261 \\
\hline & 77)Brás Cubas & 100.000 \\
\hline & 78)Caieiras & 95.780 \\
\hline & 79)Cajamar & 71.805 \\
\hline & 80)Campo Limpo Paulista & 81.693 \\
\hline & 81)Carapicuíba & 394.465 \\
\hline & 82)Cotia & 229.548 \\
\hline & 83)Embu das Artes & 264.448 \\
\hline & 84)Embu Guaçu & 67.296 \\
\hline & 85)Ferraz de Vasconcelos & 186.808 \\
\hline & 86)Francisco Morato & 169.942 \\
\hline
\end{tabular}




\begin{tabular}{|c|c|c|}
\hline \multirow{3}{*}{ 10) Franco da Rocha/SP } & 87)Franco da Rocha & 147.650 \\
\hline & 88)Guararema & 28.344 \\
\hline & 89)Guarulhos & 1.337 .087 \\
\hline \multirow{13}{*}{$\begin{array}{l}\text { 11) Itapecerica da } \\
\text { Serra/SP }\end{array}$} & 90)Sede & 152.380 \\
\hline & 91)Juquitiba & 30.642 \\
\hline & 92)São Lourenço da Serra & 15.323 \\
\hline & 93)Itapevi & 226.488 \\
\hline & 94)Itaquaquecetuba & 356.774 \\
\hline & 95)Jandira & 120.177 \\
\hline & 96)Osasco & 696.382 \\
\hline & 97)Poá & 112.917 \\
\hline & 98)Salesopolis & 16.688 \\
\hline & 99)Santa Isabel & 54.926 \\
\hline & 100)Suzano & 282.441 \\
\hline & 101)Taboão da Serra & 275.948 \\
\hline & 102)Vargem Grande Paulista & 48.720 \\
\hline \multirow[t]{9}{*}{ 12)Santos/SP } & 103)Sede & 434.359 \\
\hline & 104)Bertioga & 56.555 \\
\hline & 105)Cubatão & 126.105 \\
\hline & 106)Guarujá & 311.230 \\
\hline & 107)Itanhanhem & 97.439 \\
\hline & 108)Mongaguá & 52.492 \\
\hline & 109)Peruíbe & 63.815 \\
\hline & 110)Praia Grande & 304.705 \\
\hline & 111)São Vicente & 357.989 \\
\hline \multirow[t]{7}{*}{ 13)Taubaté/SP } & 112)Sede & 305.174 \\
\hline & 113)Monteiro Lobato & 4.467 \\
\hline & 114)Tremembé & 44.912 \\
\hline & 115)Pindamonhangaba & 162.327 \\
\hline & 116)Roseira & 10.344 \\
\hline & 117)Caçapava & 91.162 \\
\hline & 118)Redenção da Serra & 3.879 \\
\hline
\end{tabular}




\begin{tabular}{|c|c|c|}
\hline & 119)Lagoinha & 4.954 \\
\hline & 120)São Luís do Paraitinga & 10.404 \\
\hline \multirow[t]{6}{*}{ 14)Jacareí/SP } & 121)Sede & 226.539 \\
\hline & 122)Jambeiro & 6.092 \\
\hline & 123)Santa Branca & 13.770 \\
\hline & 124)Guararema & 28.344 \\
\hline & 125)Santa Isabel & 54.926 \\
\hline & 126)Igaratá & 9.349 \\
\hline \multirow[t]{20}{*}{ 15)Campinas/SP } & 127)Sede & 1.173.370 \\
\hline & 128)Americana & 231.621 \\
\hline & 129)Artur Nogueira & 51.126 \\
\hline & 130)Cosmópolis & 67.960 \\
\hline & 131)Engenheiro Coelho & 19.059 \\
\hline & 132)Holambra & 13.698 \\
\hline & 133)Hortolândia & 219.039 \\
\hline & 134)Indaiatuba & 235.367 \\
\hline & 135)Itatiba & 114.912 \\
\hline & 136)Jaguariúna & 53.069 \\
\hline & 137)Monte Mor & 56.335 \\
\hline & 138)Morungaba & 13.085 \\
\hline & 139)Nova Odessa & 57.504 \\
\hline & 140)Paulínia & 100.128 \\
\hline & 141)Pedreira & 46.094 \\
\hline & 142)Santa Barbara d'Oeste & 191.024 \\
\hline & 143)Santo Antonio de Posse & 22.597 \\
\hline & 144)Sumaré & 269.522 \\
\hline & 145)Valinhos & 122.163 \\
\hline & 146)Vinhedo & 73.855 \\
\hline \multirow[t]{4}{*}{ 16) Piracicaba/SP } & 147)Sede & 391.449 \\
\hline & 148)Águas de São Pedro & 2.707 \\
\hline & 149)Capivari & 53.152 \\
\hline & 150)Charqueada & 15.085 \\
\hline
\end{tabular}




\begin{tabular}{|c|c|c|}
\hline & 151)Elias Fausto & 17.241 \\
\hline & 152)Mombuca & 3.456 \\
\hline & 153)Rafard & 9.030 \\
\hline & 154)Rio das Pedras & 33.464 \\
\hline & 155)Saltinho & 7.919 \\
\hline & 156)Santa Maria da Serra & 5.962 \\
\hline & 157)São Pedro & 3.205 \\
\hline \multirow[t]{7}{*}{ 17)Jundiaí/SP } & 158)Sede & 401.896 \\
\hline & 159)Cambreuva & 46.528 \\
\hline & 160)Campo Limpo Paulista & 80.847 \\
\hline & 161) Itupeva & 54.128 \\
\hline & 162)Jarinu & 27.473 \\
\hline & 163)Louveira & 43.862 \\
\hline & 164)Várzea Paulista & 116.601 \\
\hline \multirow[t]{18}{*}{ 18)Ribeirão Preto/SP } & 165)Sede & 674.405 \\
\hline & 166)Altinópolis & 16.199 \\
\hline & 167)Barrinha & 31.579 \\
\hline & 168)Batatais & 64.040 \\
\hline & 169)Brodowski & 23.780 \\
\hline & 170)Cajuru & 25.455 \\
\hline & 171)Cassia dos Coqueiros & 2.607 \\
\hline & 172)Cravinhos & 34.384 \\
\hline & 173)Dumont & 9.325 \\
\hline & 174)Guariba & 38.861 \\
\hline & 175)Guatapará & 7.496 \\
\hline & 176)Jaboticabal & 76.196 \\
\hline & 177)Jardinópolis & 42.358 \\
\hline & 178)Luiz Antônio & 13.703 \\
\hline & 179)Mococa & 68.897 \\
\hline & 180)Monte Alto & 49.721 \\
\hline & 181)Morro Agudo & 31.923 \\
\hline & 182)Nuporanga & 7.299 \\
\hline
\end{tabular}




\begin{tabular}{|c|c|c|}
\hline & 183)Orlândia & 42.996 \\
\hline & 184)Pitangueiras & 38.554 \\
\hline & 185)Pontal & 46.818 \\
\hline & 186)Pradópolis & 20.169 \\
\hline & 187)Sales Oliveira & 11.541 \\
\hline & 188)Santa Cruz da Esperança & 2.097 \\
\hline & 189)Santa Rita do Passa Quatro & 27.546 \\
\hline & 190)Santa Rosa de Viterbo & 25.869 \\
\hline & 191)Santo Antônio da Alegria & 6.785 \\
\hline & 192)São Simão & 15.165 \\
\hline & 193)Serra Azul & 13.516 \\
\hline & 194)Serrana & 43.293 \\
\hline & 195)Sertãozinho & 121.412 \\
\hline & 196)Taiuva & 5.604 \\
\hline & 197)Tambaú & 23.241 \\
\hline & 198)Taquaral & 2.821 \\
\hline \multirow[t]{7}{*}{ 19)Vitória/ES } & 199)Sede & 359.555 \\
\hline & 200)Vila Velha & 479.664 \\
\hline & 201)Cariacica & 384.621 \\
\hline & 202)Viana & 75.652 \\
\hline & 203)Serra & 494.109 \\
\hline & 204)Fundão & 20.376 \\
\hline & 205)Guarapari & 121.506 \\
\hline \multirow[t]{9}{*}{ 20)Barbacena/MG } & 206)Sede & 135.829 \\
\hline & 207)Alfredo Vasconcelos & 6.709 \\
\hline & 208)Antônio Carlos & 11.112 \\
\hline & 209)Bia Fortes & 3.648 \\
\hline & 210)Desterro do Melo & 30.026 \\
\hline & 211)Ibertioga & 51.053 \\
\hline & 212)Piedade do Rio Grande & 4.703 \\
\hline & 213)Ressaquinha & 4.848 \\
\hline & 214)Santa Barbara do Tugúrio & 8.852 \\
\hline
\end{tabular}




\begin{tabular}{|c|c|c|}
\hline & 215)Santa Rita do Ibitipoca & 3.580 \\
\hline & 216)Santana de Garambeu & 2.235 \\
\hline & 217)Senhora dos Remedios & 10.604 \\
\hline \multirow[t]{8}{*}{ 21)Betim/MG } & 218)Sede & 412.003 \\
\hline & 219)Contagem & 648.766 \\
\hline & 220) Ibirité & 171.932 \\
\hline & 221)Juatuba & 25.490 \\
\hline & 222)Mario Campos & 14.811 \\
\hline & 223)Sarzedo & 30.478 \\
\hline & 224)Igarapé & 34.851 \\
\hline & 225)São Joaquim de Bicas & 25.537 \\
\hline \multirow{9}{*}{$\begin{array}{l}\text { 22)Conselheiro } \\
\text { Lafaiete/MG }\end{array}$} & 226)Sede & 126.420 \\
\hline & 227)Casa Grande & 2.309 \\
\hline & 228)Catas Altas da Noruega & 3.652 \\
\hline & 229)Cristiano Otoni & 5.215 \\
\hline & 230)Itaverava & 5.723 \\
\hline & 231)Lamim & 3.504 \\
\hline & 232)Queluzito & 1.953 \\
\hline & 233)Rio Espera & 5.876 \\
\hline & 234)Santana dos Montes & 3.892 \\
\hline \multirow[t]{5}{*}{ 23)Ipatinga/MG } & 235)Sede & 259.324 \\
\hline & 236)Santana do Paraiso & 32.232 \\
\hline & 237)Itabira & 118.481 \\
\hline & 238)Timóteo & 88.255 \\
\hline & 239)Coronel Fabriciano & 109.857 \\
\hline \multirow[t]{5}{*}{$\begin{array}{l}\text { 24)Ribeirão das } \\
\text { Neves/MG }\end{array}$} & 240)Sede & 325.846 \\
\hline & 241)Esmeraldas & 68.133 \\
\hline & 242)Pedro Leopoldo & 63.406 \\
\hline & 243)Vespasiano & 120.510 \\
\hline & 244)São José da Lapa & 22.592 \\
\hline 25)Sete Lagoas/MG & 245)Sede & 234.231 \\
\hline
\end{tabular}




\begin{tabular}{|c|c|c|}
\hline & 246)Baldim & 7.913 \\
\hline & 247)Cachoeira da Prata & 3.716 \\
\hline & 248)Fortuna de Minas & 2.913 \\
\hline & 249)Funilândia & 4.237 \\
\hline & 250)Inhaúma & 6.200 \\
\hline & 251)Jequitibá & 5.153 \\
\hline & 252)Santana de Pirapama & 7.997 \\
\hline \multirow[t]{21}{*}{ 26)Rio de Janeiro/RJ } & 253)Sede & 6.498 .837 \\
\hline & 254)São Gonçalo & 1.044 .058 \\
\hline & 255)Duque de Caxias & 886.917 \\
\hline & 256)Nova Iguaçu & 797.435 \\
\hline & 257)Niterói & 497.883 \\
\hline & 258)Belford Roxo & 494.141 \\
\hline & 259)São João de Meriti & 460.541 \\
\hline & 260)Magé & 236.319 \\
\hline & 261)Itaboraí & 230.786 \\
\hline & 262)Mesquita & 171.020 \\
\hline & 263)Nilópolis & 158.319 \\
\hline & 264)Maricá & 149.876 \\
\hline & 265)Queimados & 144.525 \\
\hline & 266)|taguaí & 120.855 \\
\hline & 267)Japeri & 100.562 \\
\hline & 268)Seropédica & 83.667 \\
\hline & 269)Rio Bonito & 57.963 \\
\hline & 270)Guapimirim & 57.105 \\
\hline & 271)Cachoeiras de Macacu & 56.603 \\
\hline & 272)Paracambi & 50.071 \\
\hline & 273)Tanguá & 32.703 \\
\hline \multirow[t]{4}{*}{ 27)Volta Redonda/RJ } & 274)Sede & 263.659 \\
\hline & 275)Barra do Piraí & 94.778 \\
\hline & 276)Barra Mansa & 180.126 \\
\hline & 277)Pinheiral & 24.076 \\
\hline
\end{tabular}




\begin{tabular}{|l|l|l|}
\hline & 278)Piraí & 26.314 \\
\hline & 279)Rio Claro & 17.425 \\
\hline
\end{tabular}

Tabela 3. Comarcas a serem visitadas na Região Centro-Oeste, os municípios cobertos pelas comarcas e a população residente em cada município.

\begin{tabular}{|c|c|c|}
\hline COMARCA & \multirow{2}{*}{\begin{tabular}{|l} 
MUNICÍPIO \\
280 Sede
\end{tabular}} & № HABITANTES \\
\hline \multirow[t]{6}{*}{ 28)Dourados/MS } & & 215.486 \\
\hline & 281)Itahum & 2.500 \\
\hline & 282)Panambi & 41.473 \\
\hline & 283)Inocência & 7.687 \\
\hline & 284)Diadópolis & 12.712 \\
\hline & 285)Laguna Carapã & 7.097 \\
\hline \multirow[t]{3}{*}{ 29)Várzea Grande/MT } & 286)Sede & 271.339 \\
\hline & \multirow{2}{*}{\begin{tabular}{|l}
$\begin{array}{l}\text { 287)Nossa Sra. Do } \\
\text { Livramento }\end{array}$ \\
288)Cristo Rei
\end{tabular}} & 12.518 \\
\hline & & 29.502 \\
\hline \multirow[t]{5}{*}{ 30) Anapolis/GO } & 289)Sede & 370.875 \\
\hline & 290)Anicuns & 32.614 \\
\hline & 291)Ceres & 20.924 \\
\hline & 292)Pires do Rio & 54.512 \\
\hline & 293)Rio Vermelho & 88.389 \\
\hline \multirow[t]{15}{*}{ 31)Goiânia/GO } & 294)Sede & 1.448 .639 \\
\hline & 295)Abadia de Goiás & 8.053 \\
\hline & 296)Aparecida de Goiânia & 532.135 \\
\hline & 297)Aragoiania & 9.607 \\
\hline & 298)Bela Vista de Goiás & 28.077 \\
\hline & 299)Bom Finopolis & 8.836 \\
\hline & 300)Brasabrantes & 3.565 \\
\hline & 301)Caldazinha & 3.664 \\
\hline & 302)Caturai & 5.010 \\
\hline & 303)Goianápolis & 11.460 \\
\hline & 304)Goianira & 40.338 \\
\hline & 305)Guapó & 14.462 \\
\hline & 306)Hidrolandia & 20.121 \\
\hline & 307)Inhumas & 51.932 \\
\hline & 308)Neropolis & 27.816 \\
\hline
\end{tabular}




\begin{tabular}{|l|l|l|}
\hline & 309)Nova Veneza & 9.249 \\
\hline & 310)Santo Antônio de Goiás & 5.659 \\
\hline & 311)Senador Canedo & 102.947 \\
\hline & 312)Teresópolis de Goiás & 7.513 \\
\hline & 313)Trindade & 119.385 \\
\hline
\end{tabular}

Tabela 4. Comarcas a serem visitadas na Região Norte, os municípios cobertos pelas comarcas e a população residente em cada município.

\begin{tabular}{|l|l|l|}
\hline COMARCA & MUNICÍPIO & № HABITANTES \\
\hline 32)Belem/PA & 314)Sede & 1.446 .042 \\
\hline & 315)Ananindeua & 510.834 \\
\hline & 316)Benevides & 59.836 \\
\hline & 317)Marituba & 125.435 \\
\hline & 318)Santa Barbara do Pará & 17.141 \\
\hline & 319)Santa Isabel do Pará & 67.686 \\
\hline & 320)Castanhal & 192.571 \\
\hline
\end{tabular}

Tabela 5. Comarcas a serem visitadas na Região Nordeste, os municípios cobertos pelas comarcas e a população residente em cada município.

\begin{tabular}{|l|l|l|}
\hline COMARCA & MUNICIPIO & № HABITANTES \\
\hline 33)Feira de Santana/BA & 321)Sede & 1.362 .880 \\
\hline & 322)Conceição do Jacuípe & 115.680 \\
\hline & $\begin{array}{l}\text { 323)São Gonçalo dos } \\
\text { Campos }\end{array}$ & 293.989 \\
\hline & 324)Amélia Rodrigues & 124.075 \\
\hline & 325)Conceição da Feira & 159.776 \\
\hline & 326)Tanquinho & 209.026 \\
\hline 34)Vitória da Conquista/BA & 327)Sede & 350.284 \\
\hline & 328)Iguá & 27.787 \\
\hline & 329)José Gonçalves & 11.015 \\
\hline & 330)Inhobim & 330 \\
\hline & 331)Anagé & 20.096 \\
\hline & 332)Barra do Choça & 34.788 \\
\hline & 333)Candido Sales & 27.918 \\
\hline & 334)Itambé & 23.106 \\
\hline & 335)Encruzilhada & 23.786 \\
\hline
\end{tabular}




\begin{tabular}{|c|c|c|}
\hline & 336)Ribeirão do Largo & 8.713 \\
\hline & 337)Planalto & 26.225 \\
\hline & 338)Belo Campo & 16.026 \\
\hline \multirow[t]{18}{*}{ 35)Fortaleza/CE } & 339)Sede & 2.609 .716 \\
\hline & 340)Maranguape & 123.570 \\
\hline & 341)Pacatuba & 72.299 \\
\hline & 342)Aquiraz & 77.717 \\
\hline & 343)Caucaia & 325.441 \\
\hline & 344)Maracanaú & 209.057 \\
\hline & 345)Eusébio & 51.913 \\
\hline & 346) Itatinga & 38.933 \\
\hline & 347)Guaiuba & 26.091 \\
\hline & 348)Pacajus & 61.846 \\
\hline & 349)Horizonte & 55.187 \\
\hline & $\begin{array}{l}\text { 350)São Gonçalo do } \\
\text { Amarante }\end{array}$ & 47.791 \\
\hline & 351)Pindoretama & 20.430 \\
\hline & 352)Cascavel & 70.574 \\
\hline & 353)Paracuru & 33.665 \\
\hline & 354)Paraipaba & 32.256 \\
\hline & 355)Trairi & 54.820 \\
\hline & 356)São Luís do Curu & 12.805 \\
\hline \multirow[t]{14}{*}{ 36)Juazeiro do Norte/CE } & 357)Sede & 268.248 \\
\hline & 358)Abaiara & 11.483 \\
\hline & 359)Araripe & 21.345 \\
\hline & 360)Antonina do Norte & 7.253 \\
\hline & 361)Assaré & 23.191 \\
\hline & 362)Aurora & 24.548 \\
\hline & 363)Barbalha & 59.343 \\
\hline & 364)Brejo Santo & 48.451 \\
\hline & 365)Barro & 22.361 \\
\hline & 366)Campos Sales & 27.167 \\
\hline & 367)Caririaçu & 26.876 \\
\hline & 368)Crato & 129.662 \\
\hline & 369)Farias Brito & 18.789 \\
\hline & 370)Granjeiro & 4.459 \\
\hline
\end{tabular}




\begin{tabular}{|c|c|c|}
\hline & 371)Jardim & 27.074 \\
\hline & 372)Jati & 7.827 \\
\hline & 373)Lavras da Mangabeira & 31.359 \\
\hline & 374)Mauriti & 46.335 \\
\hline & 375)Milagres & 28.292 \\
\hline & 376)Missão Velha & 35.326 \\
\hline & 377)Nova Olinda & 15.310 \\
\hline & 378)Penaforte & 8.888 \\
\hline & 379)Porteiras & 14.965 \\
\hline & 380)Potengi & 10.856 \\
\hline & 381)Salitre & 16.248 \\
\hline & 382)Santana do Cariri & 17.479 \\
\hline & 383)Tarrafas & 8.875 \\
\hline & 384)Várzea Alegre & 40.255 \\
\hline \multirow[t]{22}{*}{ 37)Imperatriz/MA } & 385)Sede & 253.873 \\
\hline & 386)Davinópolis & 12.579 \\
\hline & 387)Gov. Edison Lobão & 18.042 \\
\hline & 388)Vila Nova dos Martírios & 13.730 \\
\hline & 389)Açailândia & 110.543 \\
\hline & 390)Cidelândia & 13.593 \\
\hline & 391)São Francisco do Brejão & 11.863 \\
\hline & 392)Alto Parnaíba & 10.765 \\
\hline & 393)Amarante do Maranhão & 40.756 \\
\hline & 394)Arame & 31.702 \\
\hline & 395)Balsas & 93.511 \\
\hline & 396)Nova Colinas & 33.535 \\
\hline & 397)Fortaleza dos Nogueiras & 12.257 \\
\hline & 398)Buriticupu & 71.227 \\
\hline & 399)Bom Jesus da Silva & 33.615 \\
\hline & 400)Carolina & 23.979 \\
\hline & 401)Estreito & 41.392 \\
\hline & 402)São Pedro dos Crentes & 4.425 \\
\hline & 403)Grajaú & 60.093 \\
\hline & 404)Formosa da Serra & 17.757 \\
\hline & 405)Itaipava do Grajaú & 14.297 \\
\hline & 406)Itinga do Maranhão & 25.357 \\
\hline
\end{tabular}




\begin{tabular}{|c|c|c|}
\hline & 407)João Lisboa & 20.381 \\
\hline & 408)Montes Altos & 9.413 \\
\hline & 409)Ribamar Fiquene & 7.563 \\
\hline & 410)Sitio Novo & 15.871 \\
\hline & 411)Porto Franco & 21.530 \\
\hline & 412)Campestre do Maranhão & 13.369 \\
\hline & 413)Lajeado Novo & 6.923 \\
\hline & 414)São João do Paraíso & 11.388 \\
\hline & 415)Riachão & 20.209 \\
\hline & 416)Feira Nova do Maranhão & 8.126 \\
\hline & $\begin{array}{l}\text { 417)São Domingos do } \\
\text { Maranhão }\end{array}$ & 33.607 \\
\hline & 418)Gov. Luiz Rocha & 7.337 \\
\hline & 419)Fortuna & 15.098 \\
\hline & $\begin{array}{l}\text { 420)São Pedro da Agua } \\
\text { Branca }\end{array}$ & 12.028 \\
\hline & 421)Senador La Roque & 17.998 \\
\hline & 422)Buritirana & 14.784 \\
\hline & 423)Tasso Fragoso & 7.796 \\
\hline \multirow{17}{*}{ 38)São Luís/MA } & 424)Sede & 1.082 .935 \\
\hline & 425)Alcântara & 21.851 \\
\hline & 426)Anajatuba & 25.291 \\
\hline & 427)Barreirinhas & 61.621 \\
\hline & 428)Catanhede & 20.448 \\
\hline & 429)Pirapemas & 17.381 \\
\hline & 430)Matões do Norte & 16.552 \\
\hline & 431)Humberto de Campos & 23.189 \\
\hline & 432)Primeira Cruz & 13.954 \\
\hline & $\begin{array}{l}\text { 433)Santo Amaro do } \\
\text { Maranhão }\end{array}$ & 13.820 \\
\hline & 434)Icatu & 25.145 \\
\hline & 435)Axixa & 11.407 \\
\hline & 436)Itapecuru-Mirim & 62.110 \\
\hline & 437)Miranda do Norte & 24.427 \\
\hline & 438)Morros & 19.116 \\
\hline & 439)Cachoeira Grande & 8.446 \\
\hline & 440)Paço do Lumiar & 119.915 \\
\hline
\end{tabular}




\begin{tabular}{|c|c|c|}
\hline & 441)Raposa & 26.327 \\
\hline & 442)Rosário & 42.016 \\
\hline & 443)Bacabeira & 14.925 \\
\hline & 444)Santa Rita & 36.556 \\
\hline & 445)São Jose do Ribamar & 176.008 \\
\hline & 446)Vargem Grande & 55.710 \\
\hline & 447)Nina Rodrigues & 13.746 \\
\hline & 448)Presidente Vargas & 10.717 \\
\hline \multirow[t]{16}{*}{ 39)Caxias/MA } & 449)Sede & 161.926 \\
\hline & 450)São João do Sóter & 18.225 \\
\hline & 451)Aldeias Altas & 23.952 \\
\hline & 452)Codó & 120.548 \\
\hline & 453)Coelho Neto & 46.750 \\
\hline & 454)Afonso Cunha & 5.905 \\
\hline & 455)Duque Bacelar & 10.649 \\
\hline & 456)Coroatá & 61.725 \\
\hline & 457)Peritoró & 21.201 \\
\hline & 458)Igarapé Grande & 11.041 \\
\hline & 459)Bernardo do Mearim & 5.996 \\
\hline & 460)Poção de Pedras & 19.708 \\
\hline & $\begin{array}{l}\text { 461)São Mateus do } \\
\text { Maranhão }\end{array}$ & 39.093 \\
\hline & 462)Alto Alegre do Maranhão & 26.619 \\
\hline & 463)Timbiras & 28.575 \\
\hline & 464)Timon & 166.295 \\
\hline 40)Olinda/PE & 465)Sede & 389.494 \\
\hline \multirow[t]{10}{*}{ 41)Petrolina/PE } & 466)Sede & 326.017 \\
\hline & 467)Curral Queimado & 20.715 \\
\hline & 468)Rajada & 9.833 \\
\hline & 469)Cristália & 2.522 \\
\hline & 470)Nova Descoberta & 34.676 \\
\hline & 471)Tapera & 30.088 \\
\hline & 472)Izacolândia & 2.607 \\
\hline & 473)Pedrinhas & 8.833 \\
\hline & 474)Uruas & 2.803 \\
\hline & 475)Lagoa dos Carneiros & 615 \\
\hline
\end{tabular}




\begin{tabular}{|c|c|c|}
\hline & 476)Caatinguinha & 235 \\
\hline & 477)Caititu & 696 \\
\hline & 478)Cruz de Salinas & 397 \\
\hline & 479)Pau Ferro & 72 \\
\hline & 480)Atalho & 630 \\
\hline & 481)Caiçara & 580 \\
\hline & 482)Barreiro & 40.720 \\
\hline & 483)Capim & 700 \\
\hline & 484)Lagoa dos Cavalos & 481 \\
\hline & 485)Varginha & 21.955 \\
\hline & 486)Lagoa da Pedra & 6.989 \\
\hline & 487)Lajedo & 36.628 \\
\hline & 488)Jatobá & 14.464 \\
\hline & 489)Amargosa & 8.852 \\
\hline & 490)Aranzel & 782 \\
\hline & $\begin{array}{l}\text { 491)Angico Alto (Sítio dos } \\
\text { Moreira) }\end{array}$ & 635 \\
\hline \multirow[t]{4}{*}{$\begin{array}{l}\text { 42)Cabo de } \\
\text { Agostinho/PE }\end{array}$} & 492)Sede & 202.636 \\
\hline & 493)Santo Agostinho & 1.387 \\
\hline & 494)Juçaral & 5.784 \\
\hline & 495)Ponte dos Carvalhos & 5.688 \\
\hline \multirow[t]{5}{*}{ 43)Parnaíba/PI } & 496)Sede & 178.897 \\
\hline & 497)Luís Correia & 28.406 \\
\hline & 498)Bom Princípio do Piauí & 5.304 \\
\hline & 499)Buriti dos Lopes & 19.441 \\
\hline & 500)llha Grande & 8.734 \\
\hline \multirow[t]{9}{*}{ 44)Santa Rita/PB } & 501)Sede & 135.915 \\
\hline & 502)Capim & 5.601 \\
\hline & 503)Rio Tinto & 23.431 \\
\hline & 504)Lucena & 11.730 \\
\hline & 505)Pedras de Fogo & 27.032 \\
\hline & 506)Alhandra & 18.007 \\
\hline & 507)Conde & 21.400 \\
\hline & 508)Bayeux & 96.583 \\
\hline & 509)Cabedelo & 57.944 \\
\hline
\end{tabular}




\begin{tabular}{|l|l|l|}
\hline & 510)Sapé & 52.463 \\
\hline & 511)Cruz do Espirito Santo & 17.389 \\
\hline 45)Mossoró/RN & 512)Sede & 259.815 \\
\hline & 513)Tibau & 3.687 \\
\hline & 514)Grossos & 9.393 \\
\hline & 515)Areia Branca & 25.315 \\
\hline & 516)Serra do Mel & 10.287 \\
\hline & 517)Assu & 57.743 \\
\hline & 518)Upanema & 14.448 \\
\hline & 519)Gov. Dixd-Sept Rosado & 12.992 \\
\hline & 520)Baraúna & 26.799 \\
\hline
\end{tabular}

Fonte: Elaboração dos autores.

\section{Critérios de inclusão}

Os processos foram coletados cronologicamente do mais recente para o mais antigo. Os processos iniciados entre os anos de 2012 e 2017 deveriam ter decisão proferida por juiz de primeira instância. Por uma opção metodológica, a data da decisão e não a data da publicação foi considerada.

Não foram coletados dados referentes a processos em segunda instância ou fora do período temporal previsto (2012-2017).

\section{Dados de interesse}

Foram coletados dois conjuntos de dados de cada processo. Um conjunto que permitiam a localização e validação dos dados e um segundo conjunto de dados relativo às demandas em saúde e suas respectivas motivações e fundamentações.

Dados sobre a instrução processual foram necessários para ajudar a compreender a formação do processo e a trajetória do demandante até o momento da decisão do juiz de primeira instância. São dados que contribuem para responder sobre o cumprimento da Resolução nº 07/2009 do CNJ e sobre o tipo de representação jurídica utilizada pelo demandante. Foi possível depreender desse conjunto de dados que o demandante utiliza de representação legal de fora de seu estado de residência e/ou do local da comarca onde deu entrada na ação judicial.

Os dados referentes às demandas em saúde ajudam a compreender o tipo de solicitação que é feita, suas motivações e justificativas e, consequentemente, contribui para 
potencialmente conhecer possíveis falhas de acesso aos produtos e serviços de saúde ofertados na rede pública.

O formulário utilizado na coleta dos dados divide as variáveis em 5 grupos, o que permitem a rastreabilidade do processo. Assim, todos os dados são rastreáveis e, em última necessidade, ser recapturados para validação e conferência.

Primeiro: a natureza do processo caracterizou o processo como individual ou coletivo e identificou o réu, institucionalmente (se Secretaria Municipal de Saúde ou outros órgãos em litisconsórcio).

Segundo: autoria do processo contêm dados sobre a localização municipal da residência/domicílio do autor, sua representação legal e gênero. Não foram coletadas informações de caráter pessoal que identificasse o autor da ação, seu representante legal, profissionais de saúde que o assistiram e forneceram documentos, nem quaisquer outros atores processuais.

Terceiro: representação legal devem ser informados, os dados referentes à região do representante legal e a caracterização deste representante.

Quarto: solicitações foram coletados dados que descrevem cada solicitação, de acordo com sua categoria (medicamentos, internação, cirurgias, consultas, exames complementares, entre outros) e as condições de saúde (CID) que justificam as solicitações, as provas (laudo médico, exames ou outros documentos), e o embasamento legal do pedido (incluindo legislação específica em que o pedido esteja fundamentado).

Quinto: decisão "proferida pelo juiz" foram coletados dados sobre o seu deferimento/indeferimento, embasamento legal para a decisão, existência de penalidade ou bloqueio de verba para obrigar o cumprimento da decisão e comprovação da efetivação da demanda judicial.

\section{A Construção do Formulário}

O formulário utilizado na pesquisa sobre demandas judiciais junto aos Tribunais estaduais serviu de base para a criação de uma versão atualizada. A nova versão acrescentou questões que incluem variáveis de interesse não previstas anteriormente, como o deslocamento do demandante para obtenção de comprovação médica ou representação legal. 
As perguntas fechadas admitiam uma lista de respostas categóricas possíveis. Isso teve o objetivo de facilitar o controle de qualidade dos dados no preenchimento do formulário e o cadastro em si dos processos que, consequentemente, facilitou a disponibilização de um painel com a simulação do acompanhamento em tempo real das demandas em saúde no sistema judiciário.

Campos descritivos (com texto livre) foram acrescentados para descrições de detalhes e para expandir a categoria "outros" quando a lista fornecida para o item não contivesse a resposta adequada. Também há campo descritivo para doença do demandante e para melhor descrever as demandas da ação judicial.

\section{Coleta de dados}

Foram criadas equipes para cobrir todas as regiões do país. As equipes foram sediadas na capital de um estado de cada região. Em todas as regiões, há tribunais informatizados, o que facilitou a coleta dos dados em primeira instância.

Houve dois sistemas para a digitalização dos processos e informatização dos trâmites nos tribunais, o Sistema e-SAJ (Sistema Eletrônico de Automação da Justiça) que permite acesso aos processos de forma mais simples, exigindo do pesquisador inscrição da OAB (Ordem dos Advogados do Brasil) e comprovação de atividade profissional. O sistema PROJUDI (Processo Eletrônico do Judiciário) exige o certificado eletrônico gerado pela OAB e advogado cadastrado e em atividade profissional.

Ambos os sistemas permitiram a classificação das ações judiciais contidas nos processos e sua associação com palavras-chave: SUS, medicamento, tratamento, Secretaria de Saúde, Secretário de Saúde, Município, Secretaria Municipal de Saúde.

\section{Controle e supervisão na coleta dos dados}

As respostas fechadas diminuíram a possibilidade de divergência na digitação dos dados e das respostas às questões formuladas. Um manual de preenchimento do formulário e um treinamento para as equipes de campo foram fornecidos, garantindo o correto preenchimento do formulário.

As variáveis que possuíam hierarquia de informação foram apresentadas em questões que aparecem durante o preenchimento do formulário, de forma hierarquizada, diminuindo a probabilidade de erro de preenchimento. 
Os questionários preenchidos foram verificados para consistência e identificação de possíveis equívocos com a possibilidade de correção.

\section{Gestão dos dados}

Foi criada uma plataforma lógica e acessível via internet com acesso controlado por senha para a inserção dos dados de campo.

Cada registro acrescentado era automaticamente contabilizado no conjunto de gráficos de monitoramento cuja função foi fornecer aos coordenadores do projeto a visualização e o monitoramento da atualização do banco.

\section{Análise dos dados}

A análise dos dados foi feita com o auxílio de programas com pacotes estatísticos como o R e o Excel. As análises preliminares foram essencialmente descritivas sobre o conjunto primário de dados coletados e armazenados em banco.

\section{Limitações e transposição das limitações}

Entre as limitações do projeto, destacaram-se as que foram identificadas durante as fases de desenho, preparação e contato inicial com as comarcas.

Inerente ao processo de coleta de dados, o deslocamento geográfico foi de tal monta para uma captura censitária que a execução do projeto dentro da janela temporal e dos recursos financeiros disponíveis elevaria proibitivamente os riscos de não se completar a coleta de dados. Somado ao deslocamento, contatos prévios identificaram que haveria dificuldades em obter acesso aos processos em todas as comarcas.

Outra dificuldade encontrada foi o cálculo amostral. Uma amostra estatisticamente representativa exigiria a informação prévia da distribuição de frequências das variáveis de interesse, o que não foi encontrado até o momento da execução do projeto.

A opção metodológica adotada foi determinar um número de processos a serem coletados e distribuir essa coleta por uma amostra limitada de comarcas que possibilitasse substituição de comarcas quando o acesso a elas fosse negado. Segundo o levantamento feito pelo Fórum Nacional da Saúde (FNS), os tribunais estaduais e os regionais federais somaram, no ano de 2014, 392.921 processos com demandas por saúde (17). O trabalho de Asensi e Pinheiro (21) fez um levantamento mais restrito, em apenas 6 estados e 
encontrou um total significativamente inferior aos dados correspondentes levantados pelo FNS.

Tomando, portanto, os dados do FNS por referência e considerando a capacidade de coleta de dados estimada, chegou-se a um total de 8.400 processos cujos dados deveriam ser coletados para uma aproximação do conjunto de processos existentes no país.

Distribuindo-se os processos de forma proporcional em relação à população coberta para cada comarca sorteada, chegou-se ao número final de processos coletados, por comarca amostrada.

\section{Considerações Finais}

Além de seu potencial de cobrir uma lacuna de conhecimento importante sobre a natureza das demandas judiciais e políticas públicas de saúde, o projeto propôs o exercício da continuidade do monitoramento das demandas judiciais, que crescem dia após dia e se especializam em diversos aspectos.

Esse monitoramento contínuo de novas demandas judiciais será a base de um Observatório Nacional da Judicialização das Políticas Públicas de Saúde cujos relatórios periódicos possam subsidiar o Poder Público para intervir na dinâmica das ações judiciais. Espera-se que essa colaboração forneça dados para o Ministério da Saúde a fim de que possa melhorar o provisionamento de produtos e serviços e que os juízes tenham caminhos sistemáticos de suporte às suas decisões.

\section{Referências}

1. Fernandes AJN. Judicialização da saúde. Caderno Mídia Saúde Pública: comunicação em saúde pela paz. Belo Horizonte. 2007; 2(1):49-52.

2. Asensi FD. Indo além da judicialização: o Ministério Público e a saúde no Brasil. Rio de Janeiro: Escola de Direito do Rio de Janeiro da Fundação Getúlio Vargas, Centro de Justiça e Sociedade, 2010.

3. Machado FRS. A judicialização da saúde no Brasil: cidadanias e assimetrias. Rio de Janeiro: Programa de Pós-Graduação do Centro Biomédico, Instituto de Medicina Social, Universidade do Estado do Rio de Janeiro; 2010.

4. Medici AC. Judicialização, integridade e financiamento da saúde. Revista Diagnóstico e tratamento. 2010;15(2):81-87. 
5. Delduque MC, Marques SB. A Judicialização da política de assistência farmacêutica no Distrito Federal: diálogos entre a política e o direito. Revista Tempus Actas de Saúde Coletiva. 2011; 5(4):97-106.

6. Delduque MC, Marques SB, Ciarlini A. Judicialização das Políticas de Saúde no Brasil. Direito Sanitário em Perspectiva. Escola Superior do Ministério Público da União. 2003; 1(2):181-217.

7. Ramos RS, Gomes AMT. A judicialização da saúde pública no Brasil: um estudo de representações sociais. Revista Cuidarte. 2014; 5(2):827-836.

8. Bittencourt GB. O "Estado da Arte" da produção acadêmica sobre o fenômeno da judicialização da saúde no Brasil. Revista Cadernos Ibero-Americanos de Direito Sanitário. 2016; 5(1):102-121.

9. Damascena RS. Judicialização do acesso a medicamentos no município de Vitória da Conquista - BA: análise dos itinerários dos usuários. Vitória da Conquista: Programa de PósGraduação em Direito e Saúde, Escola Nacional de Saúde Pública Sérgio Arouca, Fundação Oswaldo Cruz, 2016.

10. Oliveira MRM, Delduque MC, Sousa MF, Mendonça AVM. Judicialização da saúde: para onde caminham as produções científicas? Revista Saúde Debate. 2015; 39(105):525535.

11. Costa LA. A Importância da criação de protocolos clínicos e a incorporação de novas tecnologias na redução da Judicialização. In: V Congresso de Farmácia Hospitalar em Oncologia do INCA. Rio de Janeiro, 2016.

12. D’Espíndula TCAS. Judicialização da medicina no acesso a medicamentos: reflexões bioéticas. Revista bioética. 2013; 21(3):438-447.

13. Figueiredo TA. Análise dos medicamentos fornecidos por mandado judicial na Comarca do Rio de Janeiro: A aplicação de evidências científicas no processo de tomada de decisão. Rio de Janeiro: Programa de Pós-Graduação em Saúde Pública, Escola Nacional de Saúde Pública Sergio, Fundação Osvaldo Cruz - Fiocruz, 2010.

14. Honorato S. Judicialização da Política de Assistência Farmacêutica: Discussão sobre as Causas de Pedir no Distrito Federal. Revista Cadernos Ibero-Americanos de Direito Sanitário. 2015; 4(3):116-127.

15. Asensi F, Pinheiro R. Judicialização da saúde e diálogo institucional: a experiência de lages (SC). Revista Direito sanitário. 2016; 17(2):48-65.

16. Conselho Nacional de Justiça. Resolução no 107 de 06 de abril de 2010. Supremo Tribunal Federal. Institui o Fórum Nacional do Judiciário para monitoramento e resolução das demandas de assistência à saúde. Disponível em: http://www.cnj.jus.br///images/atos_normativos/resolucao/resolucao_107_06042010_11102 012191858.pdf [Acesso em 8.fev.2018]. 
17. Conselho Nacional de Justiça. Relatório Anual CNJ 2014. Brasília, 2014. Disponível em:

http://www.cnj.jus.br/files/conteudo/destaques/arquivo/2015/04/59741adbf4e2cc6285766da da4a3f074.pdf [Acesso em 8.fev.2018].

18. Arruda SC. Análise sobre a judicialização da saúde no Estado de Mato Grosso no período de 2011-2012. Revista Cadernos Ibero-Americanos de Direito Sanitário. 2017; 6(1):86-111.

19. Delduque MC, Castro EV. A Mediação Sanitária como alternativa viável à judicialização das políticas de saúde no Brasil. Revista Saúde Debate. 2015; 39(105):506513.

20. Asensi FD, Pinheiro R. Judicialização da saúde no Brasil: dados quantitativos sobre saúde pública. Rio de Janeiro: UERJ/IMS/Cepesc, 2016.

21. Asensi FD, Pinheiro R. Judicialização da saúde no Brasil: dados e experiência. Brasilia: Conselho Nacional de Justiça. Rio de Janeiro, 2015.

22. Asensi FD, Pinheiro R. Judicialização da saúde no Brasil: dados quantitativos sobre saúde suplementar. Rio de Janeiro: UERJ/IMS/Cepesc, 2016.

\section{Como citar este artigo:}

Chrispim PP, Kato MY, Bugoni NP, Silva GM, Pittioni RB, Weber B. Construindo um observatório nacional da judicialização da política pública de saúde a partir dos municípios brasileiros: desenho de estudo. Revista Cadernos Ibero-Americanos de Direito Sanitário. 2018 jan./mar, 7(1):281-309. 